\title{
REGULACIÓN DE LAS TARJETAS DE CRÉDITO EN COSTA RICA
}

\section{CREDIT CARDS' CONTROL IN COSTA RICA}

\author{
Luis Alberto Chaves González*
}

RESUMEN

\begin{abstract}
El presente artículo tiene como objetivo abordar la temática de las tarjetas de crédito y las deficiencias en las políticas públicas para esa materia en Costa Rica, desde la perspectiva del Reglamento de Tarjetas de Crédito y Débito, el cual protege derechos como la confidencialidad, la discreción en el manejo de los datos y el derecho a la información; sin embargo, es limitado en cuanto a regular y promover un equilibrio del sistema financiero, que además permita proteger a los tarjetahabientes del exceso en las tasas de intereses.
\end{abstract}

PALABRAS CLAVE: COSTA RICA * REGULACIÓN DE CRÉDITO * TARJETAS DE CRÉDITO * SISTEMA FINANCIERO * POLÍTICA FINANCIERA * POLÍTICA CREDITICIA * TASAS DE INTERÉS

\section{ABSTRACT}

This paper aims to address the issue of credit cards and the deficiencies of public policy related to that subject in Costa Rica. For that purpose, we taking into consideration the perspective of Regulations of Credit and Debit Cards, that protect rights such as confidentiality, discretion in handling data and the information right; however, it is limited in terms of regulating and promoting the financial system balance which allows cardholders protection from excessive interest rates.

KEYWORDS: COSTA RICA * CREDIT CONTROL * CREDIT CARDS * FINANCIAL SYSTEM * FINANCIAL POLICY* CREDIT POLICY * INTEREST RATE

Dirección de Desarrollo Internacional Costarricense de Electricidad (ICE).

LAChaves@ice-go.cr 


\section{INTRODUCCIÓN}

El Ministerio de Economía, Industria y Comercio de Costa Rica (MEIC) promovió, mediante el Decreto 35867-MEIC, el Reglamento de Tarjetas de Crédito y Débito. Con esta norma publicada en La Gaceta nro. 62, alcance nro. 4 del 30 de marzo de 2010, pretende proteger los derechos de los consumidores y usuarios de conformidad con el numeral 46 de la Constitución Política, que prohíbe los monopolios y amparado en el artículo 32 de la Ley de Promoción de la Competencia y Defensa Efectiva del Consumidor 7472, publicada en La Gaceta nro. 14 del 19 de enero de 1995.

Una de las consideraciones más importante para emitir este Reglamento, es la utilización que se está haciendo de las tarjetas de crédito y débito, las cuales se han constituido en un medio de pago sustituto del dinero en efectivo, lo que hace cada vez más necesario garantizar la transparencia de la información para los agentes del mercado participantes, en virtud de salvaguardar sus intereses.

Mediante este reglamento se pretenden definir las reglas para la interpretación y aplicación de los artículos 32, 34 y 44 BIS de la Ley 7472 , relativos al régimen de responsabilidad, a la oferta, promoción, publicidad y a los requisitos que deben cumplir los emisores de tarjetas de crédito $y$ débito.

Este artículo abordará solo lo relativo a las tarjetas de crédito $y$ deficiencias de las políticas públicas en esa materia. Para familiarizar al lector con el tema es necesario clarificar algunos conceptos, cuya definición operativa se presenta conforme lo estipula el decreto referido.

Posteriormente, se presenta el estado de la cuestión en el país, más adelante, se trata los principales derechos que pretende proteger el Reglamento, como la confidencialidad, la protección de los datos y el derecho a la información. Seguidamente, se desarrolla la consideración de la necesidad de un equilibrio en el sistema y por último, la obligación del Estado de establecer políticas públicas regulatorias sobre las tarjetas de crédito.

\section{CONCEPTOS FUNDAMENTALES}

Las tarjetas de crédito establecen una relación entre agentes económicos de los que destacan el tarjetahabiente y el emisor, cuya relación se regula por el contrato de emisión de la tarjeta de crédito y se ampara en un límite de crédito. Sobre este límite se giran los desembolsos y que por ello, se deberá pagar un interés corriente, calculado sobre la base de una tasa de interés pactada en el contrato firmado por ambas partes y cuya forma de pago debe ser atendida conforme lo indica en el estado de cuenta, en el cual al menos se indica el principal, la tasa de interés corriente y el monto de intereses generado.

La temática reviste una serie de conceptos, de ellos se destacan algunos de los definidos en el artículo 2 del Reglamento:

a. Tarjetahabiente: usuario de la tarjeta de crédito o débito.

b. Emisor: entidad que emite o comercializa tarjetas de crédito y débito, para uso nacional o internacional.

c. Tarjeta de crédito: instrumento financiero que puede ser magnético o de cualquier otra tecnología, que acredita una relación contractual previa entre el emisor y el tarjetahabiente por el otorgamiento de un crédito revolutivo a favor del segundo, para comprar bienes, servicios, pagar sumas líquidas y obtener dinero en efectivo.

d. Contrato de emisión de tarjeta de crédito: contrato que regula las condiciones generales de un crédito revolutivo en moneda nacional o extranjera para la emisión y uso de la tarjeta de crédito, al cual se adhiere el tarjetahabiente por un plazo definido. Dicho contrato se regirá por los principios $y$ normas que regulan los contratos de adhesión'1.

1 Contrato de adhesión: convenio cuyas condiciones generales han sido predispuestas, unilateralmente, por una de las partes $y$ a las que se debe adherir en su totalidad la otra parte contratante. Definido por: Lic. Rodrigo Brenes Vargas (Juez Segundo Civil de San José). Responsabilidad civil en los 
e. Límite de crédito: monto máximo, en moneda nacional o extranjera o ambas, que el emisor se compromete a prestar al tarjetahabiente de crédito mediante las condiciones estipuladas en el contrato.

f. Estado de cuenta: resumen periódico de los cargos y transacciones originadas por la posesión y el uso de la tarjeta débito o de crédito y otras líneas de financiamiento asociadas a esta última en el marco de una relación contractual.

g. Principal: saldo de todas las transacciones realizadas mediante el uso de la tarjeta de crédito, con exclusión de los intereses o cargos adicionales provenientes de la generación o formación del mismo.

h. Tasa de interés corriente: porcentaje establecido por el emisor en el contrato por el uso del crédito, que se utilizará para el cálculo de intereses, sobre el saldo del principal.

i. Interés corriente: monto por intereses según la tasa pactada, calculados sobre el principal adeudado, sin incluir el consumo del período (Reglamento de Tarjetas de Crédito y Débito).

El Reglamento en estudio, incluye una amplia gama de conceptos, sin embargo para este artículo solo se destacan los antes indicados. Seguidamente, se presenta el tema de la desregulación del sector financiero y el estado de la cuestión en Costa Rica.

\section{DESREGULACIÓN DEL SECTOR FINANCIERO}

Ante la crisis del Estado de Bienestar, surge una corriente por:

... restaurar una suerte de Estado Liberal, bien provisto de individuos inteligentes, competitivos, "excelentes", alérgicos a esa mediocridad gris generada por la solidaridad puesta en instituciones: necesitamos — vienen a decir los críticos del Estado de bienestar-

contratos de adhesión a la luz del derecho del consumidor. En: <http://www.ulacit.ac.cr/revista/ rhombus18/DE-02.PDF> ciudadanos creativos más que solidarios, empresarios, más que ideólogos "excelentes" en sus empresas, más que dotados de buena voluntad (Cortina, 1998).

A partir de esa cosmología de mundo, las políticas públicas han ido en la dirección de desregular los mercados, el financiero y bancario no son la excepción. En la actualidad se podría afirmar que existe una ausencia de políticas regulatorias que consideren integralmente a todos los actores del mercado y que conduzcan a asegurar el equilibrio del sistema, en particular, en materia de tasas de interés para tarjetas de crédito en Costa Rica, dejando que lo defina el mercado "libremente".

En Costa Rica, las tasas cobradas en tarjetas de crédito no muestran una relación con la inflación promedio anual de los últimos nueve años, pues según datos del Banco Central de Costa Rica, del 2002 al 2010, la inflación promedio, medida por el Índice de Precios al Consumidor (IPC) ha sido de un $10,08 \%$, inclusive en el 2009 fue de 4,05\% y en el 2010 de $5,82 \%$. En el 2011, la inflación acumulada cerró en 4,74 \% (Banco Central de Costa Rica).

Por otro lado, la tasa básica pasiva promedio a diciembre del 2004 al 2011 es de un 10,43\%. En los últimos cuatro años, para el último mes del año muestra los siguientes datos: en el 2008 un 11,5\%; en el 2009 un $8,25 \%$; en el 2010 un $8,0 \%$ y en el 2011 igual que el año precedente, según se evidencia en el cuadro 1.

Así las cosas, es fácil visualizar una vulnerabilidad en los principios de racionalidad y proporcionalidad en el cobro de tasas de interés para tarjetas de crédito, que en muchos casos sobrepasan el $40 \%$ o el $50 \%$ anual, lo que conduce a una duplicación del principal (monto adeudado) al cabo de 24 meses, bajo el supuesto de que solo se pagaran los intereses de cada período.

El esfuerzo de emitir un Reglamento por parte del MEIC es limitado y desde un enfoque de análisis sistémico, se mantiene la desregulación en la fijación del precio del producto (el crédito). Las entidades financieras o emisores fijan las tasas de intereses en contratos 


\author{
CUADRO 1 \\ BANCO CENTRAL DE COSTA RICA \\ TASA BÁSICA PASIVA \\ 2004-2012
}

\begin{tabular}{|c|c|c|c|c|c|c|c|c|c|}
\hline & 2004 & 2005 & 2006 & 2007 & 2008 & 2009 & 2010 & 2011 & 2012 \\
\hline Enero & 13,5 & 14,5 & 15,25 & 10,75 & 7,25 & 11,25 & 8 & 7,75 & 8,75 \\
\hline Febrero & 13,5 & 14,75 & 15,25 & 9,75 & 5,5 & 12 & 8 & 7,5 & 9 \\
\hline Marz & 13,5 & 15 & 15,25 & 8 & 5,25 & 11,75 & 8 & 7,5 & 9,25 \\
\hline Abril & 13,5 & 15 & 15,25 & 7,5 & 4,25 & 11,5 & 7,75 & 7 & 9,5 \\
\hline Mayo & 13,5 & 15,75 & 13,75 & 7,25 & 5 & 11,25 & 8,5 & 7,25 & 10 \\
\hline Junio & 13,75 & 15,75 & 13,5 & 7,25 & 5,5 & 11 & 8,25 & 7,25 & 9,75 \\
\hline Julio & 13,75 & 15,5 & 13,75 & 7,25 & 7 & 12 & 8 & 7,25 & 10,25 \\
\hline Agosto & 14 & 15,5 & 13,75 & 7,25 & 8,5 & 11,5 & 8,25 & 7,25 & \\
\hline Septiembre & 14,25 & 15,25 & 13,5 & 7,25 & 9,25 & 11,5 & 7,5 & 7,75 & \\
\hline Octubre & 14,25 & 15,25 & 13,25 & 7 & 10,5 & 9 & 7,25 & 8 & \\
\hline Noviembr & 14,25 & 15,25 & 11,25 & 7 & 11 & 9,25 & 7 & 8 & \\
\hline Diciembre & 14,25 & 15,25 & 11,25 & 7 & 11,5 & 8,25 & 8 & 8 & \\
\hline
\end{tabular}

Fuente: Banco Central de Costa Rica, 2012.

de adhesión que el otro agente económico del sistema (los tarjetahabientes) solo pueden aceptar íntegramente o rechazar, pero no tienen ningún margen de negociación, pues no existe ningún mecanismo que permita definir un precio del crédito de equilibrio, es decir, una tasa de interés pactada en un proceso de libre negociación entre ambos participantes del sistema.

En el caso costarricense, por la crisis de las financieras en 1987, la política regulatoria se centró en la fiscalización de aspectos de administración de entidad, su estabilidad, solvencia y resultados financieros, sin importar si para ellos el ente acreedor cobra tasas de interés desproporcionadas, bajo el argumento de que la "racionalidad" del consumidor evitaría los excesos y lo que hay que hacer es educarlo, porque si se endeuda más allá de su capacidad de pago es por su propia responsabilidad y voluntad; cuando es evidente que el tema es tan complejo, que muchos consumidores, hasta confunden los conceptos de tasas activas y tasas pasivas.

Este es un problema internacional, que no ha estado en la agenda pública costarricense, su origen es producto de la globalización del sistema financiero, pues los bancos internacionales aplican las mismas prácticas abusivas y de latrocinio en todas sus subsidiarias, sucursales $y$ agencias de los mercados en los que operan, causando que la contraparte del sistema esté endeudada por largos períodos.

Para disfrazar su ganancia depredadora, hábilmente han mediatizado a la opinión pública con la creencia de que los deudores administran irresponsablemente sus deudas. Ello puede tener algo de cierto; sin embargo, el costo incremental producto de las altas tasas de interés inducen a deudas crecientes y recurrentes.

Por otro lado, al exigir la SUGEF, el registro de deudas y morosidad de todo ciudadano que tenga préstamos en el sistema, el tarjetahabiente, para evitar un mal perfil crediticio, recurre a más deuda para mantener sus obligaciones con la tarjeta de crédito al día, pero muchas veces, solo con el pago mínimo y cada día financieramente más ahogado; las implicaciones psicosociales de ello, pueden ser desarrolladas por otros especialistas de diferentes campos científicos.

\section{PROTECCIÓN DE DERECHOS PARA EL TARJETAHABIENTE}

En la relación establecida mediante el contrato de adhesión, la norma viene a establecer obligaciones $y$ a proteger algunos derechos 
para el tarjetahabiente, de los cuales, además del derecho a la información, destaca el de confidencialidad $y$ protección de datos.

El Reglamento establece en el artículo 22, la tutela al "Derecho de la Protección de Datos". Indica que los usuarios tienen derecho a la protección de los datos personales que las entidades financieras obtengan para la prestación de sus servicios.

Excluye algunos casos como excepciones, entre otros los que aplican por Ley 8204 "Ley sobre Estupefacientes, Sustancias Psicotrópicas, Drogas de uso No Autorizado, Actividades Conexas, Legitimación de Capitales y Financiamiento al Terrorismo" de 2001; la Ley 8754 "Ley Contra la Delincuencia Organizada" de 2009 y la Ley 7425 "Registro $y$ Secuestro de Documentos Privados e Intervención de Comunicaciones" de 1994.

Algo importante que destaca el Reglamento es que los datos personales con fines distintos al crédito, requieren el consentimiento expreso del tarjetahabiente $y$ debe estar en un documento aparte al contrato de adhesión; esto surge porque por su naturaleza, este tipo de contratos son estandarizados y no tienen cláusulas particulares para cada cliente, razón por la cual se requiere un documento legal adicional donde se otorga dicho consentimiento.

También la normativa establece que los usuarios de servicios financieros tienen derecho, cuando así lo requieran, de acceder a su información personal contenida en la base de datos del emisor y su fuente, así como a reclamar la inmediata rectificación como en derecho corresponda.

Si la información está desactualizada, deberá ser eliminada de las bases de datos de los emisores o de su fuente, por representar una afectación al acceso en los servicios financieros; para ello, el responsable de estos registros deberá brindar al interesado al menos una referencia del asiento o anotación, facilitándole el derecho a recabar información de la totalidad de ellos. Cuando el tarjetahabiente pague la totalidad de sus deudas, deben ser cancelados los asientos practicados en estos registros.

Se le otorga a las entidades financieras la obligación de adoptar las medidas técnicas de seguridad, que requieran las transacciones realizadas con tarjetas de crédito por medios electrónicos, a fin de asegurar la protección de datos personales.

Dentro de esa misma confidencialidad, se establece en el artículo 35 del Reglamento que:

Las entidades financieras, abogados, gestores o agencias de cobranza, para llevar adelante las gestiones de cobro, deberán hacerlo directamente con el deudor y sus fiadores. No se podrá realizar dicha gestión con personas distintas a las ya indicadas. Tampoco podrán utilizar prácticas de acoso y hostigamiento para el cobro de las acreencias.

Esto evita que personas ajenas a la deuda se vean informadas de la situación y se violente el derecho de la confidencialidad.

$\mathrm{El}$ otro derecho fundamental que tutela este Reglamento es el de la información, pues obliga al emisor de que informe al tarjetahabiente de todas las condiciones que afectan la emisión de las tarjetas y la notificación de modificaciones a los contratos. Sin embargo, al ser un contrato de adhesión el tarjetahabiente se ve obligado a aceptarlas, sin consulta previa, pues si no lo acepta, la única alternativa que tiene es devolver la tarjeta de crédito, pero ello implica cancelar el monto del principal adeudado; no obstante, si dentro de su capacidad de pago no le es posible cancelar su deuda total, no le queda otra opción que aceptar la modificación.

Un elemento positivo de la norma es que obliga a las entidades financieras a entregar un estado de cuenta con una determinada cantidad de datos mínimos, dentro de los cuales se incluye toda la información para hacer el cálculo de los intereses. El capítulo III de dicho Reglamento es amplio en definir hasta dieciocho rubros que deben obligatoriamente aparecer en el estado de cuenta, esto al menos le ofrece claridad al tarjetahabiente sobre las condiciones y el estado de su crédito.

El articulo 15 define como se deben calcular los intereses y señala que: "con el propósito de no generar intereses sobre intereses o intereses capitalizables... no podrá utilizarse el modelo geométrico". 
El objetivo básico de la regulación es proteger los derechos de información del tarjetahabiente y confidencialidad de datos. Incluso con respecto a la publicidad, el Capítulo IV regula el derecho de los usuarios de recibir una publicidad clara $y$ no engañosa, sin que se induzca a error, en cumplimiento con los principios de: Veracidad, Claridad, Legibilidad, Contraste, Alineación y Orientación del texto. Además, la misma no podrá suprimir condiciones o limitaciones determinantes para la decisión de consumo.

El Reglamento no menciona un derecho fundamental para el consumidor, que se sustenta en la "teoría del valor" y más antiguamente, en la edad media influida por la doctrina Católica, con la "teoría del precio justo"3.

La teoría del valor considera que el valor de un bien o servicio depende directamente de la cantidad de trabajo que lleva incorporado. La escuela clásica de la economía política lo denominada como teoría substantiva del valor $y$ establece que el valor es el monto necesario para la producción social de un bien económico.

El valor del dinero se expresa por la tasa de interés de mercado, para los norteamericanos la Prime Rate, para los europeos la LIBOR y para los costarricenses la Tasa Básica Pasiva (TBP).

Lo usual es que por encima de esa tasa principal, el prestamista cobre una sobretasa que equivale a varios puntos porcentuales a la tasa básica, con el cual el acreedor cubre el valor de inflación, sus costos internos y una ganancia razonable. Sin embargo, en el mercado crediticio nacional no existe políticas públicas que regulen cual es el techo o tope máximo de sobretasa, presentándose situaciones en que se cobra un precio por encima del valor real, lo que significa que los

2 Friedrich von Wieser, de la escuela austríaca, afirmaba que los factores de la producción tienen un valor según la utilidad conferida al producto final (su utilidad marginal).

Concepto tratado por Langenstein, quien se destacó notablemente en la defensa del estado corporativo. Citado por Raymond de Roover. El concepto del precio justo: teoría y política económica. En: $<$ http://www.institutoacton.com.ar/articulos/rroover/artroover2.pdf> tarjetahabientes se vean obligados a destinar más recursos de su ingreso (producto de su trabajo) para atender el pago, consecuentemente reduciendo su ingreso disponible y restringiendo a las familias su capacidad de consumo para otros bienes y servicios básicos.

\section{EL EQUILIBRIO EN EL SISTEMA}

Como ya se ha planteado, la doctrina económica detrás del modelo económico imperante, es que el mercado libremente fija las tasas de interés (precio). La lógica del modelo argumenta que para garantizar su funcionamiento, el derecho a la información se convierte en un principio básico. Para ello, el Estado Liberal debe proveer las herramientas jurídicas para que el cliente pueda comportarse racionalmente y sepa elegir entre la oferta disponible, eso es lo que el Reglamento analizado aporta a la sociedad.

Sin embargo, no todos los precios pueden fijarse en el libre juego del mercado, depende de su "elasticidad precio" y especialmente cuando los recursos de los distintos agentes económicos en juego es asimétrico. Es ahí donde se requiere la participación de un "árbitro" imparcial, rol que debe jugar el Estado.

El equilibrio en el sistema resulta fundamental en este análisis, ya que existe una evidente asimetría entre el emisor y el tarjetahabiente, en donde el usuario no tiene la posibilidad real de incidir sobre las políticas de cobro $y$ crédito que ofrece la entidad financiera, razón por la cual el papel del Estado es fundamental, como árbitro y agente equilibrador de la relación en el sistema financiero, el Reglamento es deficiente $\mathrm{u}$ omiso en este tema.

Bajo el pensamiento liberal imperante, desde la década de los 90, se eliminó la regulación del sistema, especialmente en tasas de interés y se centró la ejecución de la política pública en la vigilancia del comportamiento de los entes financieros y su sanidad operativa, siguiendo indicadores de Basilea I y II.

El Reglamento emitido por el Ministerio de Economía, Industria y Comercio (MEIC), tiene como objetivo básico el de proteger los derechos de información y confidencialidad de los datos de los consumidores, por lo que el 
fondo del problema público, relativo al equilibrio del sistema financiero a largo plazo y la asimetría entre los actores participantes en el sistema no se aborda.

Desde esa perspectiva, la regulación no legisla sobre las variables financieras que podrían causar desequilibrios en el sistema, sobre todo porque según datos del Ministerio de Economía, Industria y Comercio (MEIC) al primer trimestre de 2012, la deuda por tarjetas de crédito en Costa Rica asciende a \$594 179 millones de colones. Según el Banco Central de Costa Rica, al 2012 se estima un Producto Interno Bruto (PIB) a precios de mercado que asciende a $\$ 22637485,5$ millones de colones, eso quiere decir que la deuda en tarjetas de crédito es un 2,62\% del PIB (El Financiero, 2012).

Ello hace suponer que por falta de regulación podría afectarse al sistema y particularmente a los tarjetahabientes, al adquirir deudas que están por encima de su capacidad de pago, debido a una política muy laxa por parte de los emisores; también es necesario ejercer regulación sobre el sistema mismo y no solo sobre los indicadores financieros de los bancos, pues el excesivo endeudamiento caro (con altas tasas de interés), por encima de la capacidad de pago del deudor, puede generar cuentas impagas con impactos sobre otras variables económicas e incluso, consecuencias psicosociales que afectan la estabilidad familiar.

Según información de La Nación Digital, al 15 de febrero de 2011, el saldo que se debía en tarjetas de crédito en Costa Rica era de \$486 253 millones de colones, un año después asciende a \$594 179 millones de colones, es decir creció en un 22,19\%. Ello, aparte de evidenciar la necesidad de educar a los consumidores, también muestra la urgente necesidad de regular a los acreedores.

Según Adriana Rojas, abogada de la organización Consumidores Libres, en declaraciones a la prensa indica que: "el interés que cobran los emisores de las tarjetas en muchos casos pueden estar vulnerando el principio de racionalidad $y$ proporcionalidad, son abusivos $y$ en muchos casos también arbitrarios".

Un tarjetahabiente a pesar de que siga todos los "consejos expertos", sin embargo puede resultar víctima de las altas tasas y prácticas de crédito y cobro desreguladas, cuyo pago puede llegar a consumir una porción considerable del ingreso familiar, lo que obliga a recurrir a más endeudamiento, mediante este mismo instrumento de financiamiento $\mathrm{u}$ otro.

Por otro lado, la Superintendencia General de Entidades Financieras (SUGEF), una institución del modelo del Estado Liberal, se enfoca en la protección, desempeño y solidez financiera de los bancos. En cuanto a las metas de cumplimiento de los modelos de Basilea I y II, exige que todo deudor esté registrado en esa Superintendencia y que cualquier entidad financiera pueda consultar la condición de deuda del cliente, produciendo que aunque alguien tenga capacidad de pago, si el índice de endeudamiento no cumple con el definido por la SUGEF, no será sujeto de crédito, restringiendo con ello el acceso a otras fuentes de crédito para el deudor.

Ello, aparte del efecto psicológico, porque el analista de crédito ya lo empieza a ver con ojos de desconfianza y desdén; obliga al deudor en bastantes casos, a mantener tarjetas de crédito costosas, reduciendo las posibilidades de reconversión de pasivos o deuda caras por créditos de más largo plazo y a menor tasa de interés. Esto incide sobre la liquidez del tarjetahabiente, su capacidad de pago y podría generar una burbuja financiera en el largo plazo, que expone en riesgo financiero la solvencia del sistema mismo.

¿Qué pasará si estalla esa burbuja financiera y el índice de morosidad que exige la SUGEF a las entidades financiera sobre pasa el límite del 3\%? Ese índice, que es un indicador que divide el total de préstamos con mora legal $y$ en cobro judicial entre la cartera de préstamos directos del banco sujeto de supervisión, podría variar radicalmente ante un problema de iliquidez de los tarjetahabientes.

\section{FUNCIÓN REGULADORA DEL ESTADO}

El Estado costarricense tiene la obligación de velar por el sano funcionamiento de la economía, sus instituciones y diferentes agentes económicos, incluyendo la regulación a la especulación excesiva con alta rentabilidad, que se aproxima al concepto de usura. 
De conformidad con el artículo 50 de la Constitución Política, se establece que el Estado procurará el mayor bienestar a todos los habitantes del país, organizando y estimulando la producción y el más adecuado reparto de la riqueza ${ }^{4}$.

La Ley Orgánica del Banco Central de Costa Rica (Nro. 7558), vigente desde el 27 de noviembre de 1995, declara de interés público la fiscalización de las entidades financieras y crea la Superintendencia General de Entidades Financieras (SUGEF), bajo la figura jurídica de desconcentración máxima, dotada con autonomía administrativa ${ }^{5}$, cuya misión es velar por la solidez y estabilidad del sistema financiero costarricense. Su objetivo es:

Velar por la estabilidad, la solidez y el funcionamiento eficiente del sistema financiero nacional, con estricto apego a las disposiciones legales y reglamentarias $y$ de conformidad con las normas, directrices y resoluciones que dicte la propia institución, todo en salvaguarda del interés de la colectividad ${ }^{6}$.

\section{Dentro de sus funciones están:}

Dictar las normas generales que sean necesarias para el establecimiento de prácticas bancarias sanas [y] Dictar las normas generales $y$ directrices que estime necesarias para promover la estabilidad, solvencia y transparencia de las operaciones de las entidades fiscalizadas ${ }^{7}$.
El sistema financiero es fundamental en el desarrollo de la economía, ya que canaliza los recursos financieros desde las unidades económicas que tienen excedentes hacia las unidades económicas que demandan recursos, con el propósito de realizar transacciones comerciales o inversiones. Los primeros son los ahorrantes y los segundos los deudores o tomadores de crédito.

Los entes financieros surgen como actores o agentes económicos que intermedian entre ahorrantes $y$ deudores, por esto ganan comisiones o intereses según sea el riesgo asociado a la operación y la rentabilidad esperada en plazos determinados. El precio del crédito es la tasa de interés, como se ha explicado anteriormente.

En esa perspectiva, es tesis de este autor, que la SUGEF no solo debe velar por el desempeño $y$ la solidez de los entes financieros que supervisa, sino también por el funcionamiento del sistema financiero en forma integral, en el cual los deudores (tarjetahabientes) son la contraparte del sistema y resultan actores fundamentales en el funcionamiento del mercado.

En ese sentido, en coordinación con el MEIC, debería velar porque los tomadores de deuda, consumidores o tarjetahabientes, también puedan recibir precios razonables y proporcionales que les sean factibles de pagar y que no vaya a generar una burbuja financiera a largo plazo, similar a los títulos Subprime ${ }^{8}$ en el sistema financiero norteamericano, cuyo modelo de desregulación precisamente se exportó a los países subdesarrollados bajo la tutela e impulso del Consenso de Washington.
4 Este es un concepto del Estado de Bienestar que entra en contraposición con las políticas públicas del Estado Liberal.

Consultar en página de la SUGEF. En: <http://www.sugef.fi.cr/pagina. asp?lang $=0$ \&pagina $=$ servicios $/$ documentos/infgeneral/antecedentes/antecedentes.html>

6

Ídem.

Ídem.

El Subprime son hipotecas en Estados Unidos de alto riesgo, es un tipo especial de crédito hipotecario en especial para vivienda, orientado a clientes con solvencia dudosa, con el consecuente riesgo de impago superior al promedio del resto de créditos. Así las tasas de interés y las comisiones bancarias eran más elevadas que la de préstamos personales. Los bancos norteamericanos titulizaron bloques de hipotecas para diversificar riesgos y los vendieron entre inversionistas, generando una burbuja financiera en el sistema que estalló en el 2008. 
Las políticas públicas no son suficientemente claras como para que la SUGEF interprete que tiene como responsabilidad el velar por las prácticas de crédito para que no vayan a desequilibrar el sistema, razón por la que solo se ha enfocado hacia las práctica bancarias del buen desempeño de los entes financieros, sin valorar si estas entidades, para obtener ese resultado financiero, establecen condiciones leoninas y abusivas para los otros agentes económicos participantes en el sistema.

Lo anterior, porque los contratos de adhesión dejan a los tarjetahabientes en un estado de indefensión, particularmente cuando el principal adeudado no puede ser cancelado fácilmente, generándose una exposición de riesgo que no está siendo debidamente dimensionada, llevando el tema a la individualidad, bajo el argumento falaz que es absoluta responsabilidad personal de quien se endeuda y que por ignorancia o por necesidad, no ha sabido mantener sus finanzas en forma.

Esta tesis genera una "paradoja del modelo", pues contiene implícito una exposición de riesgo importante que no se está dimensionando como problema público, ya que ante una eventual inestabilidad macroeconómica, donde por ejemplo, el IPC se incremente en forma importante por otros factores exógenos, como el precio del petróleo o productos y servicios de consumo masivo, que derive en una disminución del ingreso disponible de las familias, existe el riesgo de no pago de las obligaciones en tarjetas de crédito, lo que expone la estabilidad del sistema financiero en su conjunto.

El Estado costarricense debe identificar como un problema público el nivel de endeudamiento en tarjetas de crédito y llevar a la arena política la discusión e incluir en la agenda pública la necesidad de promover una política integral en materia de tarjetas de crédito que permita proteger al usuario y la exposición de riesgo en el mercado financiero por las elevadas tasas de interés y alto nivel de endeudamiento, además de la necesidad de otorgarle mayor responsabilidad a la SUGEF para que actúe en la regulación sobre las entidades financieras en esa materia, eso es urgente y necesario para el aseguramiento de la estabilidad financiera del sistema en el mediano y largo plazo.

\section{CONCLUSIÓN}

El Reglamento analizado es limitado, ya que se enfoca en el derecho de información y la confiabilidad, no entra al fondo del asunto que es el exceso en el costo del financiamiento de las tarjetas de crédito, al derecho del tarjetahabiente a un "justiprecio", ni aborda el tema de los riesgos sobre la estabilidad del sistema financiero en el largo plazo.

En Costa Rica, el volumen de la deuda en este instrumento de pago y crédito es del 2,62\% del PIB, lo que implica que por su magnitud sea más importante aún, pues una situación de morosidad impactaría en forma significativa sobre el equilibrio del sistema financiero y en el campo económico y social del país.

Ante esta situación es necesaria una política pública que obligue a la Superintendencia a velar por la sanidad integral del sistema financiero y no solo de una parte de los actores: las entidades financieras. Igualmente, la gestión pública debe involucrar al MEIC, con el fin promover una política que reglamente el tema de las tasas de interés.

Es urgente la definición de políticas para reducir el riesgo del sistema, que regule las tasas de interés en tarjetas de crédito, evitando el latrocinio y la usura, pues en las condiciones actuales los tarjetahabientes (parte integral del funcionamiento del sistema financiero), se ven condenados a pagar deudas de difícil cancelación, con el consecuente efecto pernicioso sobre su economía familiar, con consecuencias psicosociales y económicas de diverso tipo.

No está claro, ante situaciones abusivas por parte de los emisores de tarjetas de crédito, a qué ente estatal recurrir para presentar denuncia, como sí ocurre por ejemplo, con la ARESEP, regulando precios y tarifas en los diferentes servicios públicos.

Además del riesgo sistémico asociado a un eventual crecimiento de moratoria en deudas impagas, repercutiría en equilibrios económicos, como la reciente crisis inmobiliaria en Estados Unidos, que detonó un enjambre de movimientos que todavía tiene sus réplicas en el mercado norteamericano y en otras latitudes. 
Con base en lo anterior, se puede concluir que es necesaria esta política pública que permita contrarrestar la asimetría entre emisor $y$ tarjetahabiente, asegurando el equilibrio en el sistema financiero ante la indudable exposición de riesgo existente.

\section{BIBLIOGRAFÍA}

Banco Central de Costa Rica. Indicadores económicos. En: < http:// indicadoreseconomicos.bccr.fi.cr $>$ [consultado el 4 de marzo, 2011].

Banco Central de Costa Rica. IPC: variación interanual. Base: julio $2006=100$. En: <http://indicadoreseconomicos. bccr.fi.cr/indicadoreseconomicos/ $\mathrm{Cuadros} / \mathrm{fr} \mathrm{mVer} \mathrm{C}$ a $\mathrm{Cu}$ a d ro . aspx?idioma $=1 \&$ CodCuadro $=280>$

Banco Central de Costa Rica. Tasa Básica Mensual. Porcentajes a fin de mes. En: <http://indicadoreseconomicos. bccr.fi.cr/indicadoreseconomicos/ $\mathrm{Cu}$ a d r os / f r m Ver Cat Cuadro. aspx? idioma $=1 \&$ CodCuadro $=592>$

Banco Central de Costa Rica. Producto Interno Bruto e Ingreso a precios corrientes. Departamento de Estadística Macroeconómica. En: $<$ http://indicadoreseconomicos. bccr.fi.cr/indicadoreseconomicos/ $\mathrm{Cu}$ a d ros / f r m Ver C a t C u a d ro. aspx?idioma $=1 \&$ CodCuadro $=\% 20184>$ [consultado en agosto, 2012].

Brenes Vargas, Rodrigo. Responsabilidad civil en los contratos de adhesión a la luz de los derechos del consumidor. En: <http:// www.ulacit.ac.cr/revista/rhombus18/ DE-02.PDF>

Cortina, Adela. Ciudadanos del mundo. Hacia una teoría de la ciudadanía. Madrid. Alianza Editorial SA, 1998: 73.

Gobierno de Costa Rica. "Reglamento de Tarjetas de Crédito y Débito. Decreto nro. 35857-MEIC". La Gaceta 62. Alcance 4. San José, Costa Rica: Imprenta Nacional, 2010 .

Mayorga, Gabriela. "Deuda en tarjetas de crédito representa el 2,6\% del PIB". La Nación Digital. 15 de febrero 2010. En: <http://wvw.nacion.com/ln_ee/2010/ febrero/15/economia_2262852.html> [consultado el 15 de febrero de 2010].

Rodríguez Valverde, Andrea. "Millón y medio de tarjetas de crédito circulan en Costa Rica”. El Financiero.com. 16 de abril de 2012. En: <http://www.elfinancierocr. com/ef_archivo/2012/abril/22/ finanzas3140481.html>

Superintendencia General de Entidades Financieras (SUGEF). Página principal. 4 de marzo 2011. En: <http://www.sugef. fi.cr $>$ [consultado el 4 de marzo de 2011]. Vargas Acuña, Gabriel. Un concepto de ensayo. Redacción de documentos científicos, informes técnicos, artículos científicos, ensayos. Escuela de Ciencias del Lenguaje, Instituto Tecnológico de Costa Rica, 1996. En: <http//www.cientec.or.cr/ concurso2/concepto.html>

Fecha de ingreso: 27/07/2011 Fecha de aprobación: 14/08/2012 